\title{
Reflex responses of paraspinal muscles to tapping
}

\author{
M R DimitriJeViC, M R GREgoric, A M SHERWOOD, AND W A SPENCER
}

From the Department of Clinical Neurophysiology, The Institute for Rehabilitation and Research, Department of Rehabilitation, Baylor College of Medicine, Houston, Texas and the Institute of Clinical Neurophysiology, School of Medicine, University of Ljubljana, Ljubljana, Yugoslavia

SUMMARY Erector spinae reflex studies in healthy subjects revealed two responses: a $12.0 \pm 1.6 \mathrm{~ms}$ latency, oligosynaptic response, and a 30 to $50 \mathrm{~ms}$ latency response with polysynaptic reflex characteristics. There was a silent period after the first and second responses. The effect of limb position, trunk, neck, postural changes, Jendrassik manoeuvre and vibration on both responses were also evaluated.

The deep muscles of the back are considered to be primary postural muscles and are involved in maintaining and regulating upright posture. The knowledge of reflex activity of these muscles, in particular with regard to their postural role, is still incomplete, with the exception of abdominal skin reflexes. The paraspinal muscles do not have an easily available tendon, and clinically it is difficult to observe the stretch reflex responses to tapping. The neurophysiological studies of reflex activity of trunk muscles are relatively few in comparison to extensive studies of limb reflexes and facial reflexes. Some postural and kinaesiological functions of deep muscles of the back have been described on the basis of electromyographic studies. ${ }^{1-3}$ Kugelberg and Hagbarth ${ }^{4}$ electromyographically recorded skin reflexes of the erector spinae muscles elicited by noxious stimulation. The phasic and tonic stretch reflex responses of these muscles can be evoked mechanically by means of tapping or by vibratory stimulation. These reflex responses were shown to be asymmetric in scoliotic patients. $^{5}$ The aim of the present study was to describe the electrophysiological characteristics of lumbar erector spinae muscle reflex responses evoked by tapping in healthy adults, and to elucidate the possible role of segmental and suprasegmental reflex mechanisms in the behaviour of these responses. We also studied the effects of postural changes and various positions

Address for reprint requests : Milan $\mathbf{R}$ Dimitrijevic, MD, Dspartment of Clinical Neurophysiology, The Institute for Rehabilitation and Research, PO Box 20095, Houston, Texas 77025 .

Aะc spted 11 April 1980 of the body, head, trunk, and upper limbs on the evoked reflex responses and on the tonic postural activity of these muscles.

\section{Material and methods}

Ten healthy subjects were used in this study, seven males and three females, ranging in age from 25 to 45 years. Tests were conducted in both the sitting and standing positions, while the subjects were asked to relax and close their eyes. For the trials conducted while sitting, the subjects sat upon a stool with their feet on the floor. During the series of standing trials, the subjects sat on the stool after every trial for a waiting period of approximately five minutes. The exact position of the head, arms, and trunk during each trial depended on the specific manoeuvre undertaken, as described below. The reference position, in which the control values were recorded, was with the head straight ahead, trunk erect, with arms by the sides.

Stimulus Mechanical displacement of the muscles was used as the test stimulus, in the form of either taps or vibration. Taps were produced by an automatic reflex hammer. The electrodynamic hammer delivered constant amplitude taps at a rate of $0 \cdot 1$, $0 \cdot 33$, and $1 \mathrm{~Hz}$, with the tap strength adjusted beforehand to elicit a submaximal tendon jerk response; the majority of manoeuvres were conducted with a 0.33 $\mathrm{Hz}$ rate. For each manoeuvre, groups of 16 taps were applied to the erector spinae muscles; a slightly oblique caudal orientation of the hammer was used, so that taps depressed and stretched the muscles with the point of application approximately $3 \mathrm{~cm}$ lateral to the L-5 vertebra.

In those trials where vibration was one of the stimuli, an air-driven motor, originally designed as an air-powered grinder, was used with an eccentric weight attached to the shaft as a source of vibration. Its frequency was $200 \mathrm{~Hz}$ with a displacement of 
approximately $3 \mathrm{~mm}$ maximum when unloaded, and somewhat less when applied to the tissue. The vibrator was applied to the muscle belly above the electrodes.

Recording and analysis techniques Two pairs of Beckman silver-silver chloride, recessed, surface electrodes werc placed on either side of, and paral'el to, the spine over the lumbar portion of the belly of the paraspinal muscles. The upper electrode, which was the reference, was approximately 2 to $4 \mathrm{~cm}$ away from the spine at the L-2 level, with the lower, or active electrode $3 \mathrm{~cm}$ below, and in line with the upper. EMG signals from the electrodes were differentially amplified through a TECA AA6 Mk III amplifier, using a bandwidth of $32 \mathrm{~Hz}$ to $32 \mathrm{KHz}$, and a gain of 2500 , with the active (caudal) electrode connected to the negative input of the amplifier. It was found that a well-defined, relatively noise-free average was obtained after only 16 responses, so this number was chosen for the remainder of the studies. The responses to each series (of 16 taps) were averaged for a period of $200 \mathrm{~ms}$ following the stimulus; a Hewlett Packard 5480 Signal Averaging Computer was used for this. The responses were simultaneously recorded on an FM magnetic tape recorder for subsequent re-analysis. In addition, three analog integrators, operating on the full-wave rectified EMG signal, were used to make quantitative estimates of the magnitude of various portions of the responses. The integration periods were selected according to the latency and duration of each individual's responses. The output of the integrators was then averaged over the 16 responses in each group. To estimate the effectiveness of the various manoeuvres in modifying the responses, the integration averages were divided by the corresponding control group response amplitudes and compared over the entire subject posulation, excluding one subject who showed excessive spontaneous fluctuation. All illustrations were via an HP 2684 Graphics Terminal after digitisation of surface EMG records.

Manceuvres A typical trial consisted of 64 taps applied to the paraspinal muscle, divided into four sets of 16 taps each. The first and last sets of 16 taps in each trial were control sets, in which the subject stood erect, with eyes closed, arms to the sides, and head straight. A total of 16 manoeuvres in various combinations constituted the middle two sets; four involving head position, four involving trunk position, and the remainder involving variations of the standing position, arm position, or vibration. Tap responses during the experimental manoeuvre set were compared with responses from the initial control set in the same trial. The head manoeuvre involved flexion and extension of the neck and rotation of the head to the left and right. The trunk similarly was flexed left, right, forward, and backward. In one trial, the arms were extended to a horizontal position in front of the subject. In another trial, a Jendrassik-type manoeuvre was conducted, in which the subject was asked to clench the fists with arms hanging free by the sides, modifying the manoeuvre to minimise any direct contraction of the erector spinae muscles or other postural influences due to changes in arm position. Vibration was simultaneously applied to the muscle belly in one trial, and responses immediately after the cessation of vibration constituted another. Finally, a comparison of the tap responses from two sitting positions, erect versus relaxed, was also mads. In one group we also used voluntary contraction, which was usually difficult to accomplish. Therefore, the subjects were trained through the use of auditory EMG feedback to achieve isometric contraction of the lumbar paraspinal muscles.

\section{Results}

Tapping the lumbar paraspinal muscles in standing subjects produced consistent, well synchronised biphasic or triphasic EMG responses with a short latency in these muscles. The typical early response (R1) began with a small negative deflection, followed by a marked positive peak, and finally by a large 0.4 to $1.0 \mathrm{mV}$ negative deflection, as shown in fig 1 . The amplitude did fluctuate with repetitive elicitation. No particular trend was evident, however, in this fluctuation. In addition to the larger response recorded ipsilaterally, a very small response of similar latency was recorded contralaterally.

The latencies of the early responses were constant during trials and across trials in the same subject. Measuring to the first deflection of the average of 16 responses while tapping at 10 second intervals in the 10 healthy subjects, we found latencies ranging from 10.0 to $15.7 \mathrm{~ms}$, with a mean of 12.0 and a standard deviation of $1.69 \mathrm{~ms}$. Measured from the peak of the positive deflection to the baseline return at the beginning of the silent period, the duration of R 1 averaged $10 \mathrm{~ms}$. Application of a series of stimulus taps at a $1 \mathrm{~Hz}$ rate did not result in

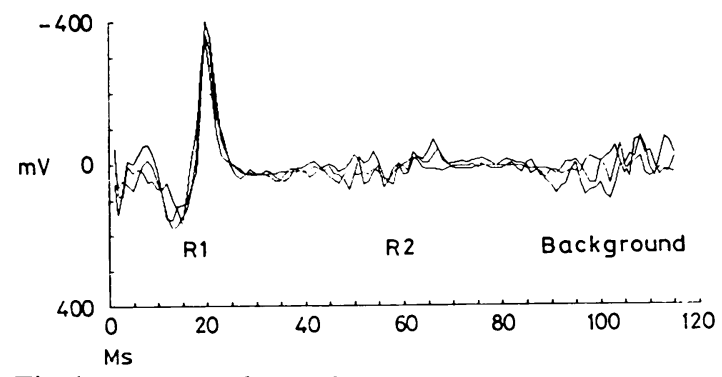

Fig 1 Paraspinal muscle response to tap. Three superimposed tap responses are shown. The early $(R 1)$ and late $(R 2)$ responses and background activity are labelled on the figure. 
marked changes in the overall response pattern, even after 160 taps. Therefore, a stimulus rate of $0.33 \mathrm{~Hz}$ was used for the remaining series of controls and manoeuvres.

At the end of the early response (R1), a period of low EMG activity occurred in most instances. This period of low EMG activity, or silent period, appeared approximately $20 \mathrm{~ms}$ after the tap and persisted for about $10 \mathrm{~ms}$ poststimulus onset. However, the characteristic background EMG activity did not resume until 80 to $200 \mathrm{~ms}$ after the tap. On some occasions, especially when the early reflex responses were of low amplitude, the silent period did not appear until 40 to $80 \mathrm{~ms}$ after the stimulus. The duration of the period of suppressed EMG activity, or so-called silent period, was very variable in individual subjects. An increase in the background activity was always accompanied by a shortening of the silent period. The silent period was also observed in recordings from the contralateral side, but it was generally of shorter duration and less complete than on the ipsilateral side. With repetitive tapping, there was a tendency toward a shorter silent period with a consequent earlier resumption of the tonic background activity, particularly when the frequency was $1 \mathrm{~Hz}$.

Following the early, synchronous response, but prior to the resumption of the background activity, one or two bursts of less synchronous EMG activity (R2) occurred, delineated by periods of relatively low EMG activity which were usually well defined. The more common observation was that one R2 burst followed the $\mathrm{R} 1$ activity prior to the resumption of the background activity. The latency of this $R 2$ response was approximatey 30 to $50 \mathrm{~ms}$. When the third burst of activity did appear, it gave the appearance of a cyclic response, since the intervals between the first and second bursts and the second and third bursts were approximately equal. The amplitude of the $R 2$ responses was usually smaller than the amplitude of the early responses, was often smaller than the tonic background activity, and in two subjects even was occasionally absent. An inverse relationship between the amplitude of the early and late responses was sometimes observed: in subjects who showed marked early responses, relatively small bursts of activity were observed, and vice versa; when the early responses were less pronounced, later responses were usually larger. Late responses were present bilaterally. The late responses on the contralateral side were of approximately the same size or larger than the ipsilateral responses (fig 2). The amplitude of

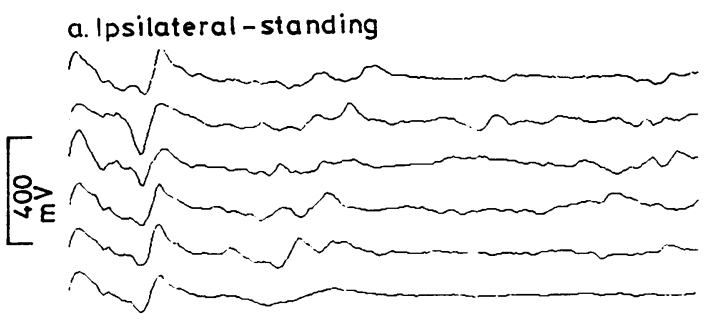

\section{b. Contralateral - standing}

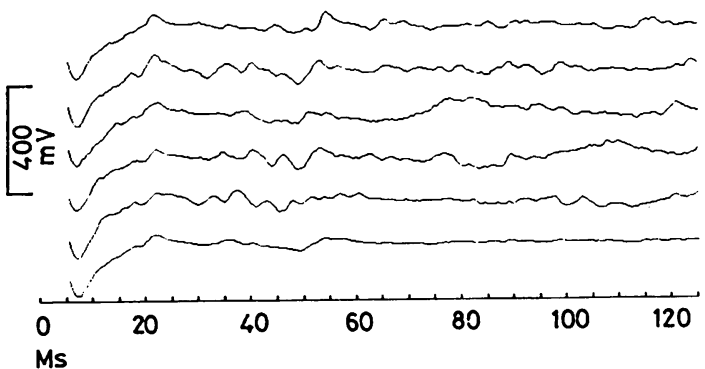

Fig 2 Ipsilateral and contralateral responses of paraspinal muscles to ipsilateral taps. A comparison of the ipsilateral and contralateral responses shows that while the $R 1$ response is present only ipsilaterally, and $R 2$ response is present bilaterally. In this and subsequent figures, five or six sequential responses are presented with traces starting at $5 \mathrm{~ms}$, to avoid stimulus artefact.

the late responses spontaneously fluctuated with repetitive taps also, but independently from the fluctuations of the early response amplitudes. Though the late responses were less constant in amplitude than the early responses, they showed no trend for consistent increase or decrease in amplitude.

The responses evoked by tapping the paraspinal muscles were well defined when the subjects were standing or sitting in an erect position. When no tonic background EMG activity was present in the paraspinal muscles, as for instance in subjects sitting in a relaxed position, the reflex responses were much smaller and often even absent (fig 3). The responses evoked in the standing position were generally larger than those evoked in the sitting position. However, in some subjects, the background activity and reflex responses were relatively small even in the standing position. In these subjects, the early response was elicited only when the trunk was slightly inclined forwards, producing an increase in both reflex responses and in background activity.

In addition to the spontaneous fluctuation in the $\mathrm{R} 1$ and $\mathrm{R} 2$ responses, and to the posturally 
a. Sitting in relaxed position

Гூ⿱宀㠯

b. Sitting in erect position

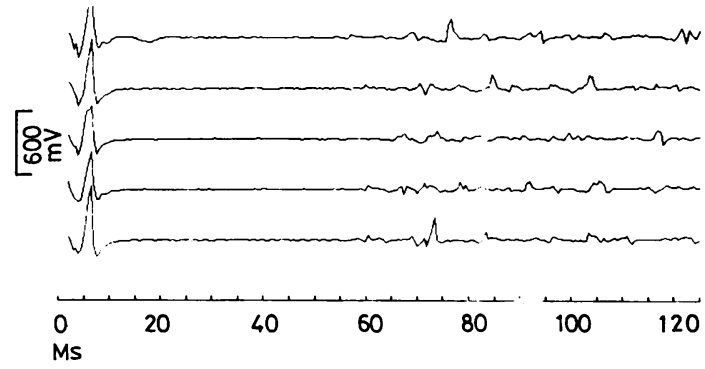

Fig 3 Paraspinal tap responses in sitting position. Erect sitting posture resulted in large, well-defined tap responses, while a relaxed posture virtually eliminated both background and tap response EMG activity.

dependent fluctuation with sitting and standing, the amplitude of the paraspinal reflex response to taps could be changed with a variety of manoeuvres as described below. The effects of the various manoeuvres on the $\mathbf{R} 1$ and $\mathbf{R} 2$ responses and on the background activity were evaluated by comparing the results obtained during the manoeuvre with those obtained in the erect standing position with arms by the side. (One subject out of the 10 was excluded because of excessive spontaneous fluctuation of the reflex responses.)

The behaviour of the evoked responses was dependent to a large extent on the position of the trunk during tapping. When the trunk was flexed forward slightly (about 15-20 degrees forward), all of the measured responses showed increases over the control values, as can be seen in fig 4. In some of the subjects, an opposite effect occurred when the degree of flexing of the trunk was increased; the evoked reflex responses and the tonic background activity decreased relative to the control values. The moderately forward flexion produced the most effective facilitation of the $R 2$ responses. The $R 1$ response was not only increased in amplitude, but was often increased in duration as well. Although accurate measurement of the latency was not readily obtained, the $\mathrm{R} 2$ response did a. Erect standing posture

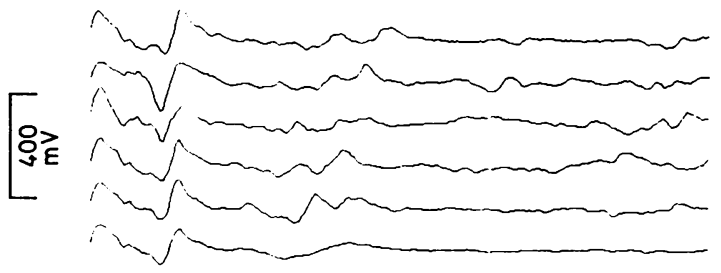

b. Trunk forward

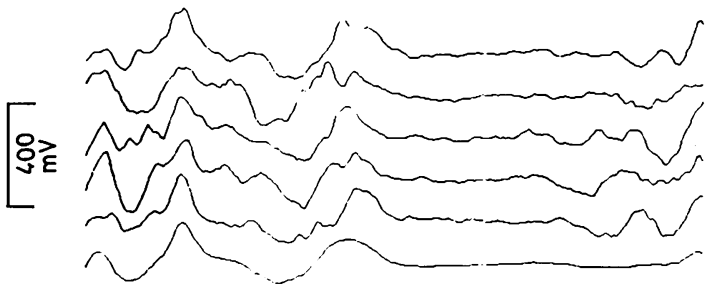

c. Trunk extended backward

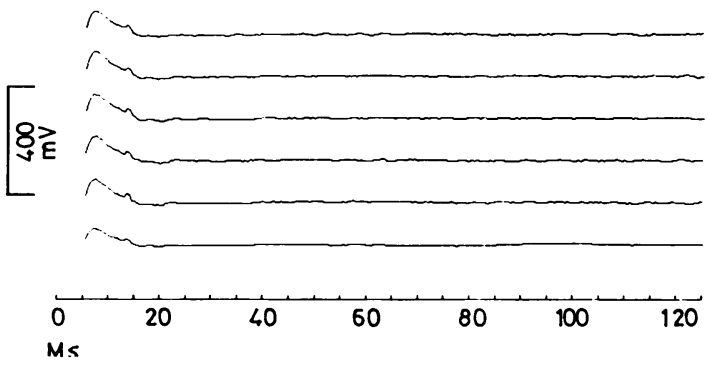

Fig 4 Paraspinal tap responses while standing. Tap responses increased when the subject changed from an erect to a slight forward-leaning posture; responses were suppressed by leaning the trunk backward.

seem more constant and of shorter latency on repetitive tapping in the flexed trunk position compared to the control series in the upright position.

The $R 1$ and $R 2$ responses were also increased with lateral movements of the trunk to the contralateral side from the tapped muscle. Although the muscle being tapped was stretched in this position, in most subjects there was no pronounced increase in the background activity. These effects were similar to the effects produced during forward flexion of the trunk.

Changes in head position affected the paraspinal reflex responses also, but to a somewhat lesser degree than changes in the trunk position. Flexing the neck had the largest effect on R1, R2, and background activity while extending the neck increased the $\mathrm{R} 2$ response only. Ipsilateral and contralateral neck flexion 
a. Control

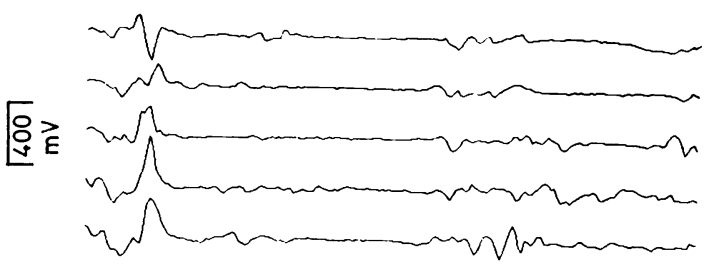

b. Tap + vibration
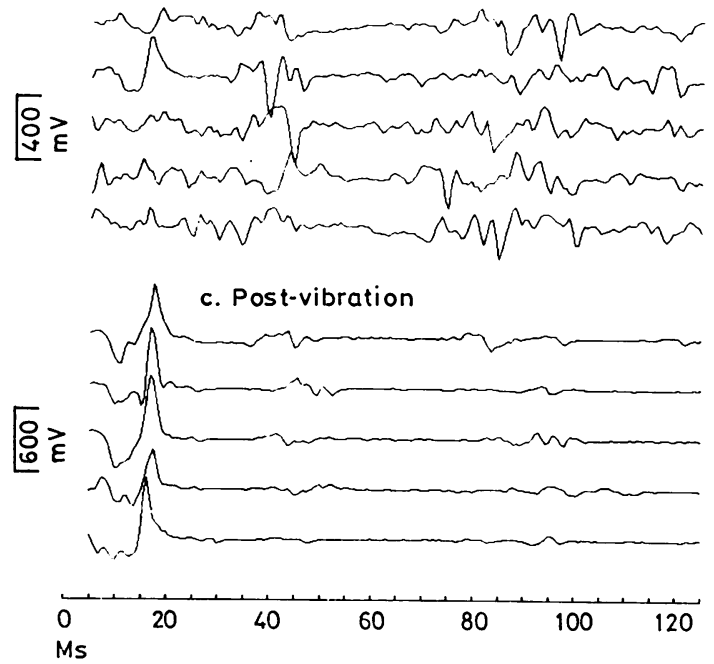

Fig 5 Paraspinal EMG response to tapping and vibration. A control scries of taps was applied before and after a series in which vibration was also applied. The results show early response $(R I)$ suppression during, and facilitation following vibration, and the opposite effect on the late response (R2). Note that part $(C)$ is plotted at a scale of 600 microvolts rather than 400 , as are (A) and $(B)$.

had little effect on any of the responses.

Interaction of phasic and tonic stretch reflexes During vibration of the paraspinal muscles and simultaneous tapping of these muscles, the amplitude of the $\mathrm{R} 1$ responses was decreased in comparison to the responses in the control series. In contrast to this, the $\mathbf{R} 2$ responses and the tonic background activity were increased during vibration as is illustrated in fig 5 . The suppression of the early responses by vibration was not clearly related to the amount of vibratory-induced tonic activity. For instance, in two subjects, there was a marked decrease in the amplitude of the early response even though vibration did not induce any tonic activity. Immediately after vibration, the $R 1$ responses were increased over the control values. The effects of this post- vibratory facilitation can also be seen in fig 5 .

The Jendrassik-type manoeuvre increased the R1 responses without significantly changing either the $\mathbf{R} 2$ reflex response or the background activity. During voluntary contraction, the $\mathrm{R} 1$ and $\mathrm{R} 2$ reflex responses and the tonic background activity were increased to approximately the same amount. Volitional activity decreased the duration of the silent period and increased the amplitude of the background activity.

\section{Discussion}

The paraspinal muscles consist of a complex group of muscles arranged in several layers and with different functions. In electromyographic analysis by means of surface electrodes placed over the paraspinal muscle bulk in the lumbar region, the recorded activity is derived mainly from the superficial layer, that is to say from the erector spinae muscles. Kinaesiological electromyographic studies of paraspinal muscles by Floyd and Silver, ${ }^{1}$ Portnoy and Morin, ${ }^{2}$ Asmussen, ${ }^{6}$ and Asmussen and Klausen ${ }^{7}$ revealed postural activity of erector spinae muscles in standing subjects and in subjects sitting in erect posture. Conversely, they also observed inactivity of these muscles when the subjects were allowed to sit in a relaxed posture. Similarly, in our studies, the behaviour of the reflex responses of these muscles evoked by tapping was related to the amount of background electromyographic activity. The responses were of very low amplitude and often absent in fully relaxed muscles, but they were increased with erect posture with concomitant tonic postural activity of the erector spinae muscles.

Kugelberg and Hagbarth ${ }^{4}$ studied spinal mechanisms of the abdominal and erector spinae skin reflexes to noxious stimulation in resting subjects; the reflex responses were only slightly increased when elicited against moderate voluntary contraction. In our study, however, the early and late responses were evoked by painless tapping more easily when erector spinae muscles were active in their primary function, (that is in sustaining the erect posture of the trunk against gravity) than when they were quiet.

The well synchronised, constant, early ipsilateral responses (R1) showed characteristics of the phasic stretch reflex: a latency consistent with the oligosynaptic reflex arc; the most effective stimulation was tapping in an oblique direction, which caused a stretch of the muscle; the $\mathrm{R} 1$ reflex response showed vibratory-induced suppression, postvibratory facilitation, and 
facilitation during a Jendrassik manoeuvre. R1 responses recorded on the contralateral side, although of much smaller amplitude, had the same latency as the ipsilateral responses. Early responses evoked on the contralateral side can be explained by propagation of the mechanical event through the tissue. ${ }^{8}$ Such responsiveness of the erector spinae muscles to mechanical stimuli applied at a distance has been shown by Trontelj, ${ }^{5}$ who showed that taps to the ligament between spinal processes of the lumbar vertebrae evoked bilateral paraspinal reflex responses.

The poorly synchronised, variable electromyographic response of longer latency (R2) showed characteristics of polysynaptic reflexes. The variable, long latency of this response suggests a polysynaptic reflex arc. The potentiation effect of vibration on $R 2$, in contrast to the suppression effect on $R 1$, supports the assumption that $R 2$ is a polysynaptic reflex. ${ }^{9}$ Futhermore, the symmetric bilateral appearance of $\mathbf{R} 2$ following unilateral stretch also supports this assumption.

The typical pattern observed after tapping was two bursts of activity separated by relative inactivity, followed by a resumption of continuous background activity. Occasionally, the reappearance of the continuous background activity was delayed by the occurence of cyclic periods of activity and inactivity. Such cyclic patterns have also been found in abdominal skin reflexes, ${ }^{10}$ in the blink reflex, ${ }^{11}$ and in the flexor reflex in normals, ${ }^{12} 13$ and in patients. ${ }^{1415}$ Pedersen, ${ }^{12}$ Faganel, ${ }^{13}$ Grimby, ${ }^{16}$ Hagbarth and Finer, ${ }^{17}$ ascribed the cyclic pattern of the flexion reflex to central mechanisms. In interpreting the results for the paraspinal reflexes, we must also consider the fact that these reflex responses are superimposed on tonic postural activity. The relative contributions of the unloading reflex and of central refractoriness in generating the silent period is uncertain, ${ }^{18}$ but in any event, supraspinal postural activity is essential in maintaining the cyclic pattern of activity.

In contrast to other polysynaptic reflexes, the R2 response did not show significant habituation after regular, repetitive stimulation even in a long series with $1 \mathrm{~Hz}$ stimuli which were neither noxious nor painful. This absence of progressive decline of the $R 2$ response during repetitive taps may be due to the presence of tonic postural activity. It has been shown for the blink reflex that voluntary contraction delays the course of habituation. ${ }^{19}$ Descending postural influences which maintain the tonic activity of erector spinae muscles and facilitate the reflex responses may have an analogous effect.

Facilitation of both $\mathrm{R} 1$ and $\mathrm{R} 2$ responses were observed with several manoeuvres: sitting erect, trunk flexed forward slightly, lateral flexing to the contralateral side, flexing the neck, voluntary contraction of the lumbar paraspinal muscles, and outstretched arms. If the standing subject stretched his arm in front of his line of balance, then an involuntary postural reaction occurred in which the position of the head, shoulders and trunk were changed in order to prevent displacement of the line of gravity. ${ }^{20}$ Erector spinae muscles have an active role in the postural reactions.

The increased excitability of the stretch reflex, as seen in the increased size of the $R 1$ response, could be due to the descending facilitation of the erector spinae muscles while the trunk is flexed. On the other hand, this could be simply explained as the effect of stretching the muscles. In contrast to marked facilitative effects of mild or moderate flexion of the trunk, a decrease in the background activity and reflex responses was observed when the trunk was further flexed. This is in agreement with the observation of Floyd and Silver ${ }^{1}$ that the erector spinae are inactive in extended forward flexion of the trunk. The changes in the early reflex responses on lateral flexion of the trunk-an increase on the ipsilateral side and a decrease on the contralateral side-can be explained as a result of stretching the muscle on the contralateral side and shortening it on the ipsilateral side.

Hagbarth and Kugelberg ${ }^{10}$ reported an increase in the size and a shortening of the latency of the trunk skin reflexes during voluntary contraction. In their studies, the erector spinae skin reflex evoked at rest was slightly increased by a moderate voluntary contraction. During voluntary contraction while standing, we found an equal increase in $R 1, R 2$, and background activity. The different effects of volitional and postural activation of erector spinae muscles on the reflex responses reflect the two kinds of organisation of descending influences on segmental reflexes.

Support for the hypothesis of different neural influences on the $\mathrm{R} 1$ and $\mathrm{R} 2$ reflexes comes also from observations of the effects of neck, limb position and vibrational reflexes. Relatively selective facilitation of the $R 2$ response occurred during neck flexion and to some extent during arm extension, as well as during vibration. Selective facilitation of the phasic stretch response (R1) occurred immediately after vibrating (postvibratory potentiation) as well as during 
Jendrassik-type manoeuvre (clenching fists with arms hanging free at the sides of the body). Delwaide $^{21}$ showed that even a passive change in the position of the upper limbs influences the excitability of the lower limbs' tendon reflexes. However, the manoeuvre used here did not involve changes in limb position.

In summary, tapping the erector spinae muscles evoked early (R1) and late (R2) reflex responses. The $\mathrm{R} 1$ response show characteristics of a phasic, oligosynaptic reflex response. Both responses were easily elicited when tonic postural activity of erector spinae muscles was present, while standing or sitting with erect posture. The behaviour of reflex responses was shown to be related to postural and antigravity functions of these muscles. A pronounced facilitation of evoked reflex responses was observed during postural changes of the trunk and upper limbs.

The authors are grateful to Dr Janez Faganel and Dr Donald Lehmkuhl for their assistance with review and critical comments. Support for this work was generously provided by The Bob and Vivian Smith Foundation, Houston, Texas, the Rehabilitation Research and Training Center No 4 (Rehabilitation Services Administration Grant 16-P-56813-6), Rehabilitation Services Administration Grant 13-P-59275-6, and Research Council of Slovenia Grant 306-1208/79.

\section{References}

1 Floyd WF, Silver PHS. The function of the erectores spinae muscles in certain movements and postures in man. $J$ Physiol 1955; 129: 184-203.

2 Portnoy H, Morin F. Electromyographic study of postural muscles in various positions and movements. Am J Physiol 1956; 186:122-6.

3 Basmajian JV. Muscles Alive: Their Functions Revealed by Electromyography. Baltimore: Williams and Wilkins, 1978; 175-88.

4 Kugelberg E, Hagbarth KE. Spinal mechanisms of the abdominal and erector spinae skin reflexes. Brain 1958; 81:390-404.

5 Trontelj JV, Pecak F, Dimitrijevic MR. Segmental neurophysiological mechanisms in scoliosis. J Bone Joint Surg 1979; 61-B(3):310-3.

6 Asmussen E. The weight-carrying function of the human spine. Acta Orthop Scand 1960; 29: 276-90.

7 Asmussen E, Klausen K. Form and function of the erect human spine. Clin Orthop 1962; 25:
$55-63$.

8 Lance JW, de Gail P. Spread of phasic muscle reflexes in normal and spastic subjects. $J$ Neurol Neurosurg Psychiatry 1965; 28:328-34.

9 Delwaide PJ. Human monosynaptic reflexes and presynaptic inhibition. In: Desmedt JE, ed. New Developments in Electromyography and Clinical Neurophysiology, Basel: Karger 1973; 3:508-23.

10 Hagbarth KE, Kugelberg E. Plasticity of the human abdominal skin reflexes. Brain 1958; 81: 305-18.

11 Gregoric M. Neurophysiological characteristics of human supraspinal interneurone system in health and disease. Thesis (in Slovene). University of Ljubljana Medical Faculty, 1976.

12 Pedersen E. Studies of the central pathway of the flexion reflex in man and animal. Acta Psychiatr Scand 1954; Suppl 88:1-81.

13 Faganel J. Electromyographic analysis of human flexion reflex components. In: Desmedt JE, ed. New Developments in Electromyography and Clinical Neurophysiology. Basel: Karger, 1973; 3:730-4.

14 Kugelberg E. Demonstration of $\mathrm{A}$ and $\mathrm{C}$ fibre components in the Babinski plantar response and the pathological flexion reflex. Brain 1948; 71:304-19.

15 Lavric A. Study of motor activity in patients with chronic capsular hemiplegia. Thesis (in Slovene). University of Ljubljana Medical Faculty, 1974.

16 Grimby L. Normal plantar response: integration of flexor and extensor reflex components. $J$ Neurol Neurosurg Psychiatry 1963; 26:39-50.

17 Hagbarth KE, Finer BL. The plasticity of human withdrawal reflexes to noxious skin stimuli in lower limbs. In: Moruzzi G, Fessard A, Jasper $\mathrm{HH}$, ed, Progress in Brain Research, Brain Mechanisms, Amsterdam, Elsevier, 1963; 1: 65-81.

18 Shahani BT, Young RR. Studies of the normal human silent period. In: Desmedt JE, ed, New Developments in Electromyography and Clinical Neurophysiology, Basel: Karger, 1973; 3: 589-602.

19 Desmedt JE, Godaux E. Habituation features of exteroceptive suppression and exteroceptive reflexes in normal man and the effect of voluntary innervation. Brain Res 1976; 106:21-9.

20 Martin JP. The Basal Ganglia and Posture. London: Pitman Medical, 1967; 127-42.

21 Delwaide PJ, Figiel C, Richelle S. Effects of postural changes of the upper limb on reflex transmission in the lower limb. Cervicolumbar reflex interactions in man. $J$ Neurol Neurosurg Psychiatry 1977; 40:616-21. 